\title{
Heartbeat: Rapid rule-out pathways for acute myocardial infarction
}

doi:10.1136/heartjnl-2020-317428

Catherine M Otto

Clinical decision making in patients presenting to the Emergency Department with chest pain remains a challenge; while only $10 \%$ to $20 \%$ are ultimately diagnosed with acute myocardial infarction (AMI), it is important to not miss any patient with an AMI. The European Society of Cardiology (ESC) 0/1 algorithm allows rapid triage of chest pain patients based on the change in high-sensitivity cardiac troponin (hs-cTn) over the first hour after presentation, dividing patients into rule-out, observation and rule-in categories. However, there appears to be some variation in the accuracy of this approach across different population cohorts and there is little data on outcomes in the rule-out and observation categories. To better understand the accuracy and prognostic value of the ESC $0 / 1$ algorithm globally, Chiang and colleagues ${ }^{1}$ performed a systematic review and meta-analysis that identified that 10 cohorts with over 11000 patients -- about $18 \%$ were assigned to rule-in, $27 \%$ to observation and $55 \%$ to rule-out categories, depending on the specific c-Tn assay. The pooled sensitivity of the ESC $0 / 1$ algorithm for AMI diagnosis was over $98 \%$ with a mortality rate in the rule-out category of only $0.1 \%(95 \% \mathrm{CI}=0.0 \%$ to $0.4 \%)$ at 30 days and $0.8 \% \quad(95 \%$ $\mathrm{CI}=0.5 \%$ to $1.2 \%$ ) at 1 year. However, the mortality rate for patients in the observation group was $0.7 \%(95 \% \mathrm{CI}=0.3 \%$ to $1.2 \%)$ at 30 days and increased to $8.1 \%$ $(95 \% \mathrm{CI}=6.1 \%$ to $10.4 \%)$ at 1 year, similar to the mortality rate in the rule-in group.

In an editorial, Chapman and Mills ${ }^{2}$ summarise these data (figure 1) and conclude: "While many emergency departments will already have implemented rapid rule-out pathways for acute myocardial infarction, some have not, particularly those in the USA. There is a plethora of data evaluating high-sensitivity troponin testing in patients with suspected acute coronary syndrome, including two prospective, randomised controlled trials, demonstrating very low rates of future myocardial infarction or death as far as 1 year after index presentation. It is time to take advantage of these strategies to

Correspondence to Professor Catherine M Otto, Division of Cardiology, University of Washington, Seattle, Washington, USA; cmotto@uw.edu
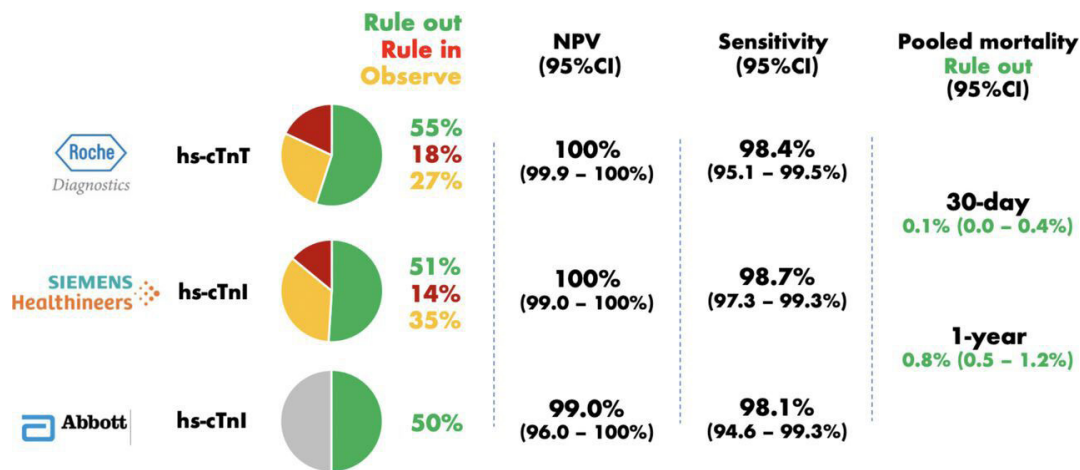

Rule out
(95\%CI)

$98.4 \%$

$(\mathbf{9 5 . 1}-\mathbf{9 9 . 5 \% )}$

30-day

$0.1 \%(0.0-0.4 \%)$

$98.7 \%$

$(\mathbf{9 7 . 3}-\mathbf{9 9 . 3} \%)$

1-year
$0.8 \%(0.5-1.2 \%$

$98.1 \%$

$(94.6-99.3 \%)$

Figure 1 Comparison of the performance of the European Society of Cardiology (ESC) Congress 0/1 hour algorithm for the rule out of index myocardial infarction across three high-sensitivity cardiac troponin assays. note no meta-estimates were obtained for either rule-in or observational zone performance using the Abbott hs-cTnl assay due to insufficient study numbers. NPV, negative predictive value. reduce overcrowding, mitigate the associated risks of hospitalisation and prevent harm.”

Stopyra and colleagues ${ }^{3}$ compared two other rapid rule-out pathways for AMI in 5788 patients presenting to the emergency department at three sites in the USA. The
History ECG Age Risk factor Troponin (HEART) Pathway classified 38\% of patients as low-risk and only $0.4 \%$ of these low risk patient had a major adverse cardiac event (MACE) at 30 days; a negative predictive value of $99.6 \%$. In comparison, the Emergency Department Assessment of

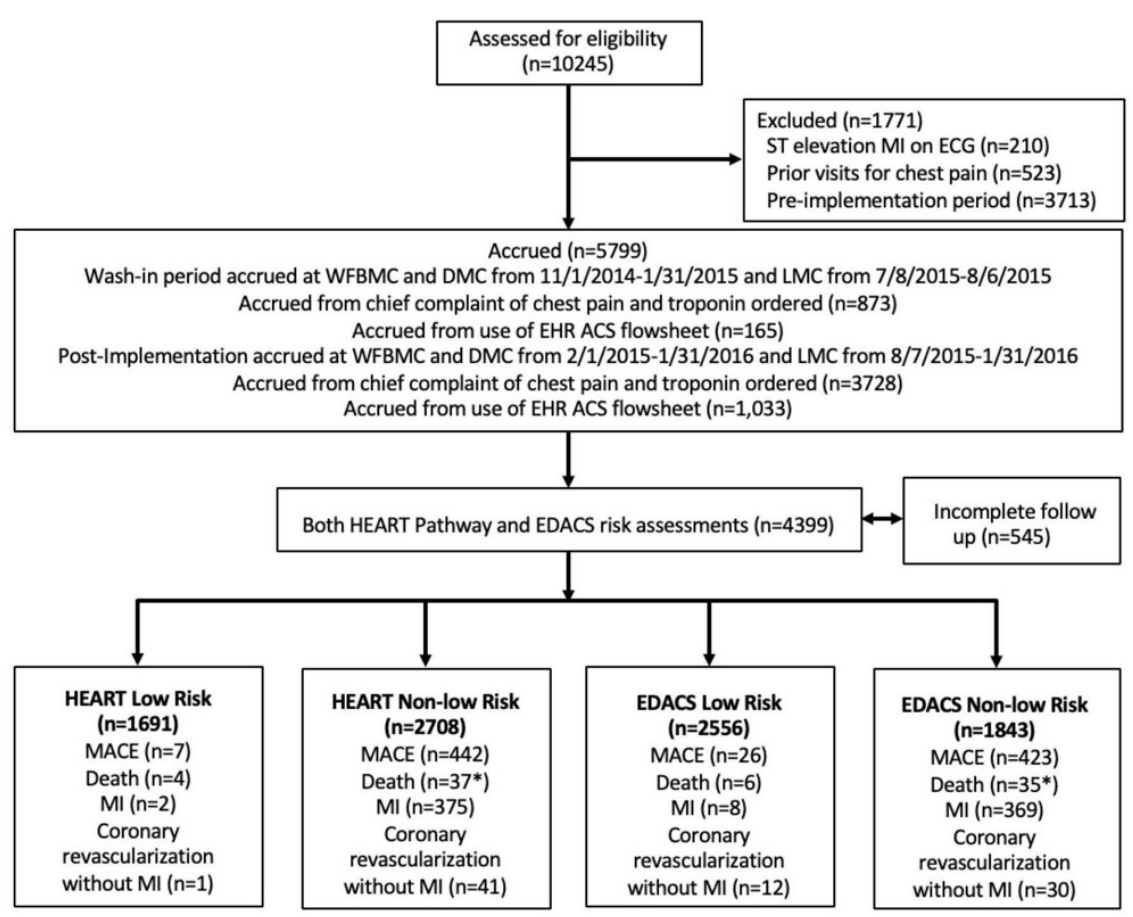

Figure 2 Flow diagram. MI, Myocardial Infarction; WFBMC, Wake Forest Baptist Medical Centre; DMC, Davie medical Centre; LMC, Lexington medical Centre; EHR, electronic health records; ACS, acute coronory syndrome; HEART, history ECG age risk factor troponin; EDACS, Emergency Department Assessment of Chest pain Score ; MACE, Major Adverse Cardiac Events. * Deaths not exclusive of MI or coronory revascularisation events. 


\section{The diagnostic impact of CMR}

Referral diagnosis

CMR diagnosis

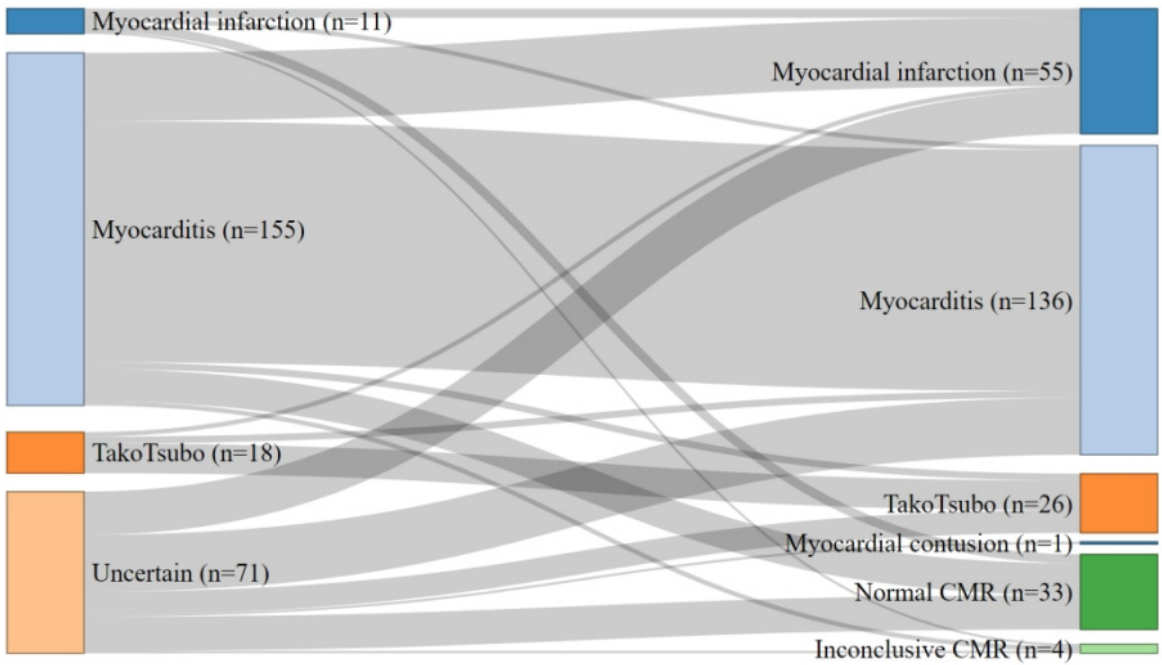

Figure 3 The diagnostic impact of early CMR. Among patients with troponin-positive acute chest pain and nonobstructed coronary arteries, an early CMR ( $\leq 7$ days) established a diagnosis in $86 \%$ of the patients. CMR confirmed the referral diagnosis in $48 \%$ and overrode it in $16 \%$, identified the aetiology in $22 \%$, revealed a structurally normal heart in $13 \%$ and remained inconclusive in $1 \%$ of the patients.

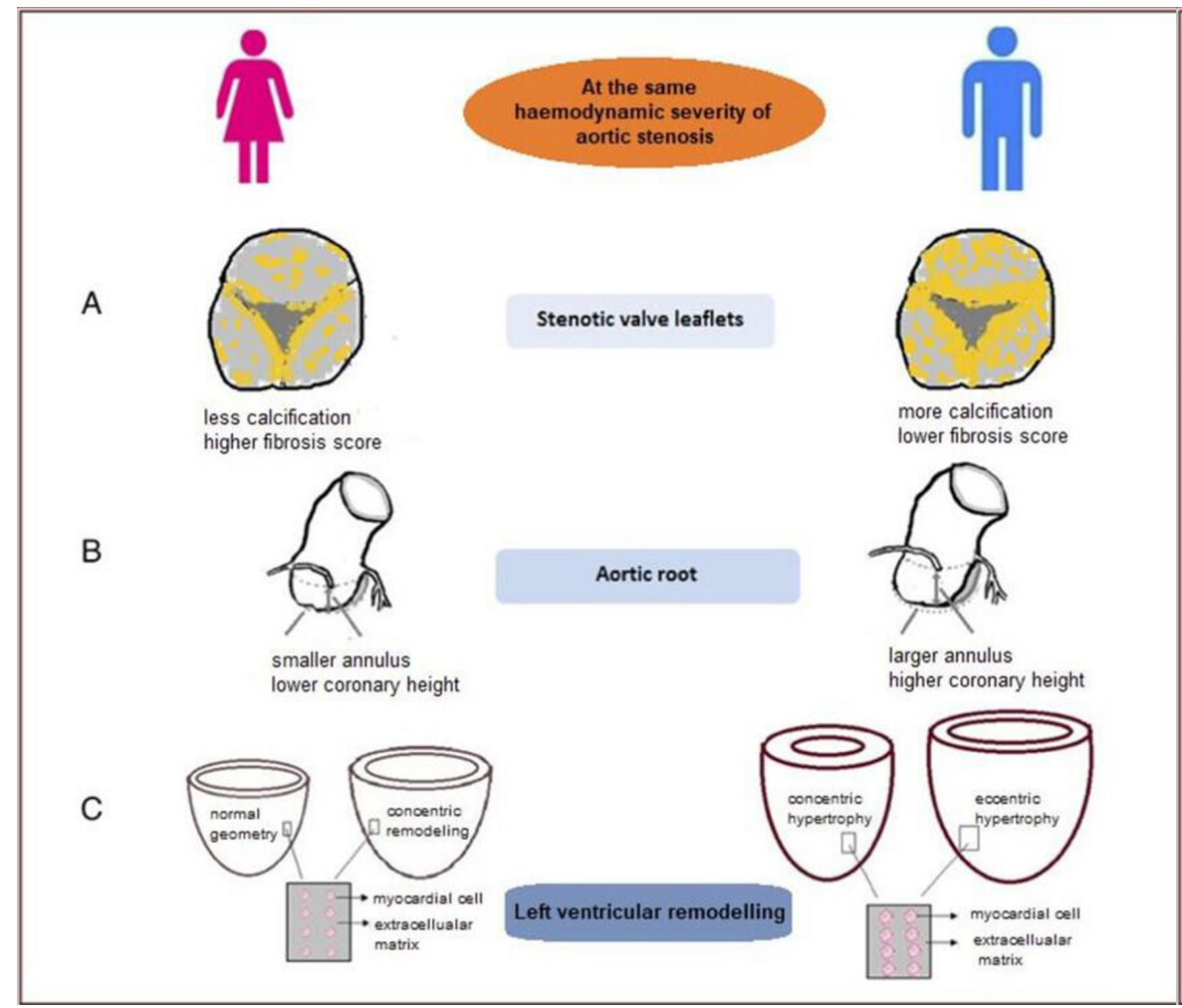

Figure 4 Summary of pathophysiological alteration of stenotic valve leaflets, aorta root and left ventricular remodelling pattern in women vs men. (A) sex differences in aortic value calcification and fibrosis. women have less AV calcification and higher fibrosis score than men for a give mean gradient or indexed aortic value area by Doppler echocardiography. (B) sex differences in aortic root dimensions between women and men. (C) with CMRI, women often exhibited lower LV mass with a smaller LV cavity size, whereas men developed a larger LV cavity, greater LV wall thickness and mass for similar as severity. as, aortic stenosis; CMRI, cardiac MRI.
Chest pain Score (EDACS) classified 58\% of patient as low risk but the 30 day MACE in these patients was $1.0 \%$ yielding a slightly lower negative predictive value of $99.0 \%$. (figure 2) The authors conclude: "EDACS identifies a larger proportion of patients as low-risk than the HEART Pathway, but has a higher missed major adverse cardiac events rate at 30 days."

Professor Brody comments ${ }^{4}$ that "Crowding of our hospitals and emergency departments (EDs) is not a new issue, but the COVID-19 pandemic has now taken it to another level. The recent pressures highlight the importance of sparing vital inpatient resources for those who really need them." Clearly we should use rapid triage approaches to the patient with chest pain but "Should you use the 1 hour algorithm, the HighSensitivity Troponin in the Evaluation of patients with suspected Acute Coronary Syndrome (High-STEACS) algorithm or should you incorporate clinical features by adopting the HEART pathway or Emergency Department Assessment of Chest Pain (EDACS) decision aid?” While there is no simple answer to balancing accuracy vs efficiency, "It is also important to consider the patient's perspective. There is evidence that patients who are allowed to engage in shared decision-making with their clinician are more likely to choose to leave the hospital without further investigations than those who receive standard, clinician-guided care."

An additional challenge in patients presenting with chest pain is management of those who have a myocardial infarction but no obstructive coronary disease (MINOCA). In a series of 255 patients with MINOCA, Vago and colleagues ${ }^{5}$ report that cardiac magnetic resonance imaging (CMR) soon (at a mean of 2.7 days) after presentation was diagnostic in $86 \%$-- myocarditis in 54\%, myocardial infarction in $22 \%$, and takotsubo cardiomyopathy in $10 \%$. (figure 3) Furthermore, the patients with a normal CMR had a 4 year mortality of $0 \%$.

A state-of-the art review article in this issue of Heart ${ }^{6}$ discusses the clinical role of advanced left ventricular imaging in adults with aortic stenosis and how parameters such as global longitudinal strain on echocardiography, late-gadolinium enhancement on CMR, exercise hemodynamics and serum biomarkers are being utilised in ongoing clinical trials of earlier intervention for aortic stenosis. Another review article by Shan and Pellikka ${ }^{7}$ discusses the distinctive characteristics of aortic stenosis in women and how these sex differences 
might impact diagnosis, management and clinical outcomes. (figure 4)

The Education in Heart article in this issue, ${ }^{8}$ outlines the causes and epidemiology of non-bacterial thrombotic endocarditis (NBTE), discusses the pathophysiology, and emphasises key principles in diagnosis and management. As the authors summarise: "NBTE is a rare condition associated with autoimmune disease, malignancy and hypercoagulable states, and characterised by sterile friable vegetations composed of fibrin and platelets with high risk of systemic embolism. Most patients are asymptomatic and the condition is commonly diagnosed at post-mortem. A high index of clinical suspicion is therefore required for diagnosis. Management requires differentiation of NBTE from infective endocarditis, definition of the underlying cause, assessment of valve lesions with echocardiography and prevention of systemic embolism by means of anticoagulant treatment. Surgery is rarely indicated, except in patients with haemodynamic compromise, valve failure or high risk of embolism."

Funding The authors have not declared a specific grant for this research from any funding agency in the public, commercial or not-for-profit sectors.

Competing interests None declared.

Patient and public involvement Patients and/or the public were not involved in the design, or conduct, or reporting, or dissemination plans of this research.

Patient consent for publication Not required.

Provenance and peer review Commissioned; internally peer reviewed.

(C) Author(s) (or their employer(s)) 2020. No commercial re-use. See rights and permissions. Published by BMJ.

\section{(D) Check for updates}

To cite Otto CM. Heart 2020;106:951-953.

Heart 2020;106:951-953.

doi:10.1136/heartjnl-2020-317428

ORCID iD
Catherine M Otto http://orcid.org/0000-0002-05279392

\section{REFERENCES}

1 Chiang C-H, Chiang C-H, Lee GH, et al. Safety and efficacy of the European Society of cardiology 0/1-hour algorithm for diagnosis of myocardial infarction: systematic review and meta-analysis. Heart 2020;106:985-91.

2 Chapman AR, Mills NL. High-Sensitivity cardiac troponin and the early rule out of myocardial infarction: time for action. Heart 2020;106:955-7.

3 Stopyra J, Snavely AC, Hiestand B, et al. Comparison of accelerated diagnostic pathways for acute chest pain risk stratification. Heart 2020;106:977-84.

4 Body R. Accuracy or efficiency? striking the balance for your chest pain pathway. Heart 2020;106:954.

5 Vágó H, Szabó L, Dohy Z, et al. Early cardiac magnetic resonance imaging in troponin-positive acute chest pain and non-obstructed coronary arteries. Heart 2020;106:992-1000.

6 Calin A, Mateescu AD, Popescu AC, et al. Role of advanced left ventricular imaging in adults with aortic stenosis. Heart 2020;106:962-9.

7 Shan Y, Pellikka PA. Aortic stenosis in women. Heart 2020;106:970-6.

8 Hurrell H, Roberts-Thomson R, Prendergast BD. NonInfective endocarditis. Heart 2020;106:1023-9. 